\title{
Atividade biológica de metabólitos secundários de algas marinhas do gênero Laurencia
}

\author{
Fernanda L. da S. Machado, ${ }^{1,2}$ Carlos R. Kaiser, ${ }^{2,3}$ Sônia S. Costa, ${ }^{4}$ Lísia M. Gestinari, ${ }^{1}$ \\ Angélica R. Soares ${ }^{*}, 1,2$
}

\author{
${ }^{1}$ Núcleo em Ecologia e Desenvolvimento Sócio-Ambiental de Macaé, Universidade Federal do Rio de Janeiro, Caixa \\ Postal 119.331, Rua Rotary Club s/n, Bairro São José do Barreto, Macaé, 27901-000 Rio de Janeiro-RJ, Brasil, \\ ${ }^{2}$ Programa de Pós-graduação em Química, Instituto de Química, Universidade Federal do Rio de Janeiro, \\ 21941-590 Rio de Janeiro-RJ, Brasil, \\ ${ }^{3}$ Laboratório de Ressonância Magnética Nuclear, Universidade Federal do Rio de Janeiro, 21941-590 \\ Rio de Janeiro-RJ, Brasil, \\ ${ }^{4}$ Laboratório de Química de Produtos Naturais Bioativos, Núcleo de Pesquisas de Produtos Naturais, \\ Universidade Federal do Rio de Janeiro, 21941-902 Rio de Janeiro-RJ, Brasil
}

\begin{abstract}
RESUMO: As algas vermelhas do gênero Laurencia são conhecidas como uma riquíssima fonte de metabólitos secundários. As principais classes químicas já isoladas são terpenos - sesquiterpenos, diterpenos, triterpenos - e acetogeninas. Estudos apontam que estas substâncias apresentam importantes atividades biológicas, principalmente citotóxica e antibacteriana. Existem ainda alguns poucos trabalhos com informações sobre atividade antiparasitária, antiviral e antifúngica dos metabólitos de Laurencia. O forte perfil bioativo observado em alguns estudos sugere que alguns destes metabólitos podem ser futuramente utilizados como fármacos ou como protótipos de novos agentes antitumorais e antibacterianos. Muitos dos metabólitos de Laurencia ainda não foram avaliados sob o ponto de vista farmacológico, o que representa um grande potencial a ser explorado por diversos campos da farmacologia.
\end{abstract}

Unitermos: macroalgas, Ceramiales, Rhodomelaceae, produtos naturais.

\begin{abstract}
Biological activity of the secondary metabolite from marine algae of the genus Laurencia." The red alga of the genus Laurencia is known as an endless source of metabolites. The main substances produced are sesquiterpenes, diterpenes, triterpenes and acetogenins. Many studies reveal that these metabolites are active, especially cytotoxic and antibacterial. There are few other studies that describe the antiparasital, antifungal and antiviral actions of the metabolites produced by the alga of the genus Laurencia. The results observed in some studies suggest that these metabolites may be used in the future as drugs or lead compounds as antitumoral and/or antibacterial agents. Many metabolites have not been submitted to pharmacological studies which still represents an area to be explored.
\end{abstract}

Keywords: macroalgae, Ceramiales, Rhodomelaceae, natural product.

\section{INTRODUÇÃO}

O ambiente marinho, principalmente nas regiões tropicais, apresenta uma diversidade de espécies comparável àquela presente nas florestas tropicais. Esta riqueza de espécies é capaz de produzir uma enorme variedade de estruturas químicas com um potencial elevado para descoberta de novos fármacos (Molinski et al., 2009).

Nas últimas três décadas, a descoberta de metabólitos com atividade biológica a partir de macroalgas cresceu substancialmente (Smit, 2004). As algas vermelhas estão entre as principais produtoras de metabólitos secundários no ambiente marinho. Dentre as espécies mais estudadas mundialmente, encontram-se as algas do gênero Laurencia (Ceramiales, Rhodomelaceae) (Cardozo et al., 2007). O gênero é encontrado em mares subtropicais e tropicais de todo mundo, abrangendo cerca de 150 espécies. No Brasil, ocorrem cerca de trinta espécies sendo três delas consideradas endêmicas, $L$. catarinenses, $L$. oliveirana e L. translucida (Algaebase; Algamare, 2008).

A pesquisa de metabólitos secundários do gênero Laurencia foi iniciada na década de 60 por Irie et al. (1965). Desde então, mais de 500 substâncias foram isoladas (De Carvalho et al., 2006). Entretanto, a pesquisa neste gênero ainda possibilita a descoberta de novas 
moléculas com diferentes padrões estruturais (Blunt et al., 2007). Os principais metabólitos já isolados de algas do gênero são sesquiterpenos (Wright et al., 1991), diterpenos (Rochfort \& Capon, 1996), triterpenos (Souto et al., 2002) e acetogeninas (Iliopoulou et al., 2002a), podendo ou não conter halogênios em sua estrutura.

A incorporação de halogênios à estrutura destes metabólitos é catalisada por haloperoxidases, sendo a vanádio-haloperoxidase a enzima de maior prevalência entre algas produtoras de metabólitos halogenados (CarterFranklin \& Butler, 2004).

Acredita-se que a produção de metabólitos secundários esteja relacionada à adaptação da alga ao ambiente marinho. Estudos mostram que os metabólitos de Laurencia podem apresentar múltiplas funções, como proteção contra herbívoros e organismos incrustantes, aumentando a capacidade adaptativa do indivíduo ao ambiente (Pereira et al., 2003). A mediação destes metabólitos em interações entre organismos pode indicar que estas moléculas também atuem em outros sistemas biológicos. Desta observação parte a motivação para a busca de moléculas de interesse terapêutico. Muitos estudos têm verificado diferentes ações farmacológicas de metabólitos de algas do gênero Laurencia (Konig et al., 1994). Este trabalho busca estimar a extensão e a relevância dos estudos já realizados que comprovem alguma ação farmacológica de metabólitos produzidos por estas algas.

O levantamento apresentado foi feito através da base de dados "Web of Science" e compreende os principais artigos publicados entre o período de 19862010 com informações sobre atividade biológica de metabólitos de Laurencia, com ênfase especial aos estudos de atividade farmacológica, como citotóxica, antitumoral, antibacteriana, antifúngica, antiparasitária e antiviral.

\section{Atividade antitumoral}

A pesquisa de novos antitumorais é uma das principais áreas estudadas atualmente no mundo. Diversas instituições de pesquisas têm concentrado seus esforços na busca de novos fármacos eficazes e seguros a serem utilizados no tratamento do câncer. Mohmammed e colaboradores publicaram em 2004 um trabalho sobre os efeitos de um diterpeno isolado de Laurencia intricata sobre células tumorais em condição de hipóxia.

$\mathrm{O}$ rápido crescimento de tumores pode superar a capacidade de vasos sanguíneos suprirem algumas áreas com oxigênio e nutrientes. As células tumorais se adaptam para sobreviverem nestas condições tornando-se mais resistentes ao tratamento por radio e quimioterapias. Geralmente, esse quadro é um indicativo de estágio avançado da doença. O principal fator transcricional responsável pela ativação da expressão gênica em condições de hipóxia é o HIF-1.

O extrato lipídico de Laurencia intricata de uma população da Jamaica apresentou atividade inibidora do fator transcricional HIF-1. O fracionamento guiado por bioensaio deste extrato levou ao isolamento do diterpeno laurediterpineol (1). Em células T47D (linhagem de células tumorais de mama), o laurediterpineol inibiu os fatores HIF-1 e o angiogênico VEGF induzidos por hipóxia e suprimiu a sobrevivência de células tumorais nestas condições. Ainda não existem fármacos que atuem especificamente neste tipo de célula. Desta forma, inibidores de HIF-1 podem representar uma potencial classe de agentes antitumorais (Dixon et al., 2007).

Inúmeros trabalhos têm apontado os efeitos citotóxicos de metabólitos de Laurencia isolados de espécies coletadas em vários locais do mundo (Rashid et al., 1995). Os principais resultados encontram-se resumidos na Tabela 1.

Tabela 1. Atividade citotóxica de metabólitos isolados do gênero Laurencia.

\begin{tabular}{|c|c|c|c|}
\hline Espécies & Substâncias & Linhagens de células sensíveis & Referência \\
\hline L. obtusa & $\begin{array}{l}2,3 \\
6,7 \\
26\end{array}$ & $\begin{array}{l}\text { K562, MCF7, HeLa, A431 } \\
\text { MCF7, PC3, HeLa, A431, K562, } \\
\text { P-388 }\end{array}$ & $\begin{array}{l}\text { Kladi et al., } 2006 \\
\text { Iliopoulou et al., } 2003 \\
\text { Takeda et al., } 1990\end{array}$ \\
\hline \multirow[t]{2}{*}{ L. microcladia } & $\begin{array}{l}4 \\
5\end{array}$ & $\begin{array}{l}\text { K562, MCF7, HeLa, A431 } \\
\text { K562, MCF7, HeLa, A431, } \\
\text { NSCLC-N6 }\end{array}$ & $\begin{array}{l}\text { Kladi et al., } 2006 \\
\text { Kladi et al., } 2006\end{array}$ \\
\hline & 8,9 & NSCLC-N6, A549 & Kladi et al., 2005 \\
\hline L. majuscula & 10 & Painel de células do National Cancer Institute & Erickson et al., 1995 \\
\hline \multirow[t]{5}{*}{ L. viridis } & 11,14 & P-388, A-549, HT-29, MEL-28 & Norte et al., 1997 \\
\hline & 11 & T47D, ZR-75-1, Hs578T, MDA-MB-231 & Pec et al., 2003 \\
\hline & 12 & P-388 & Manriquez et al., 2001 \\
\hline & 13 & P-388, A-549, HT-29, MEL-28 & Norte et al., 1996 \\
\hline & $15,16,17,18$ & P-388, A-549, HT-29, MEL-28 & Manriquez et al., 2001 \\
\hline L. tristicha & 19 & A-549, BGC-823, Bel 7402, HCT-8, HeLa & Sun et al., 2005 \\
\hline L. cartilaginea & $20,21,22,23,24,25$ & P-388, A-549, HT-29, MEL-28 & Juagdan et al., 1997 \\
\hline L. catarinensis & $27-40$ & HT-29, MCF7, A431 & Lhullier et al., 2010 \\
\hline
\end{tabular}


Sesquiterpenos isolados de Laurencia obtusa e L. microcladia, coletadas na Grécia, apresentaram atividade citotóxica contra cinco linhagens de células tumorais humanas. Das substâncias testadas, a substância 2, juntamente com 7-hidroxilaurano (3), isoladas da fração apolar do extrato de Laurencia obtusa e laurinterol (4) e bromolaurenisol (5) de Laurencia microcladia apresentaram maior atividade citotóxica. Os ensaios de citotoxidade foram feitos em células do tipo K562 (linhagem de células de leucemia crônica mielogênica), MCF7 (derivadas de adenocarcinoma mamário), HeLa (adenocarcinoma de cérvice) e A431 (carcinoma epidermóide). Em todas as linhagens de células testadas, o 7-hidroxilaurano (3) apresentou maior potência citotóxica. A atividade foi mais marcante contra células do tipo MCF7 com $\mathrm{IC}_{50} 15,8 \mu \mathrm{M}$. A linhagem K562 apresentou menor sensibilidade a esta substância, com $\mathrm{IC}_{50} 64,2 \mu \mathrm{M}$.

O bromolaurenisol (5) apresentou ainda atividade citotóxica contra a linhagem de células NSCLC-N6, proveniente de tumores pulmonares com IC50 de apenas
26,5 $\mu \mathrm{M}$ (Kladi et al., 2006).

Em outro estudo com espécimes coletadas na Grécia, isolou-se uma série de diterpenos bromados de L. obtusa. Das substâncias isoladas, o prevezol B (6) e o neorogioldiol B (7) foram as substâncias que apresentaram maior atividade citotóxica. As linhagens PC3 (originária de próstata) e HeLa, mostraram-se as mais sensíveis ao neorogioldiol B (7), com valores de IC50 de 50,8 e $34,4 \mu \mathrm{M}$ respectivamente. O prevezol B (6) foi o mais potente nas linhagens A431 e K562. Os valores de IC50 foram 65,2 e $76,4 \mu \mathrm{M}$, respectivamente (Iliopoulou et al., 2003).

Sesquiterpenos do tipo cupareno (8 e 9) isolados de uma população de Laurencia microcladia da Grécia foram citotóxicos para linhagens de células tumorais de pulmão (NSCL-N6 e A549). Comparando a estrutura destes derivados com outros não citotóxicos relacionados, verificou-se que a presença do átomo de bromo não parece afetar significativamente a atividade na classe de substâncias testadas (Kladi et al., 2005).

$\mathrm{O}$ sesquiterpeno majapoleno A (10), principal<smiles>CC1=CC(O)C(C(C)CC[C@H]2C(C)[C@]3(C)CCC2(C)O3)CC1</smiles><smiles>Cc1ccc([C@]2(C)CC/C(=C\Br)[C@H]2C)c(O)c1</smiles>

4<smiles>C=C(C)[C@H]1CC[C@@](C)(O)[C@H](C)[C@@H]1CC(C)C1CC[C@@](C)(O)[C@H](Br)C1</smiles><smiles>CC1(C)CC(C23CC(C)(C)C(C)(CCO2)OO3)CCC1Br</smiles>

10<smiles>CC1=CC[C@]2(C)C(=C(C)[C@H](O)[C@@H](Br)[C@@H]2C)CC1</smiles><smiles>Cc1cc(O)c([C@]2(C)CCC(=CBr)[C@@H]2C)cc1Br</smiles><smiles>Cc1ccc(C2=CCC(C)C2(C)C)cc1Br</smiles>
8<smiles>Cc1ccc(C2(C)CCC(C)C2C)cc1O</smiles>
9

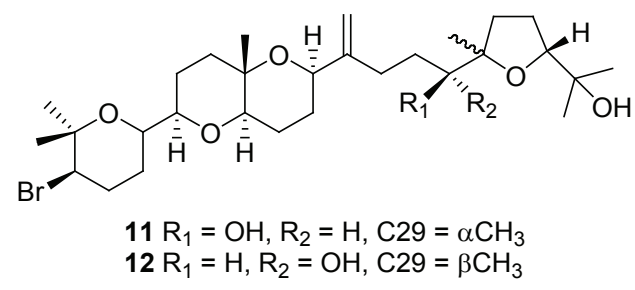

$11 \mathrm{R}_{1}=\mathrm{OH}, \mathrm{R}_{2}=\mathrm{H}, \mathrm{C} 29=\alpha \mathrm{CH}_{3}$ $\mathrm{R}_{1}=\mathrm{H}, \mathrm{R}_{2}=\mathrm{OH}, \mathrm{C} 29=\beta \mathrm{CH}_{3}$<smiles>C=C(CC[C@H]1O[C@@H](C(C)(C)C)CCC1(C)C)[C@H]1CC[C@H]2O[C@H](C3(C)CCC(Br)C(C)(C)O3)CC[C@@]2(C)O1</smiles> 


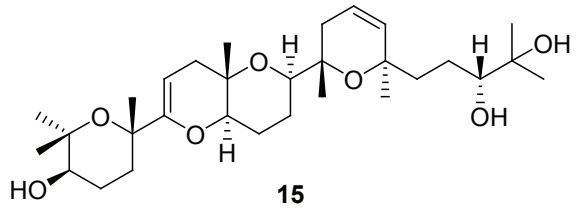

15<smiles>C=C(CC[C@@H](O)[C@]1(C)CC[C@H](C(C)(C)O)O1)C1CC[C@H]2O[C@@]3(C)CC[C@H](Br)C(C)(C)O[C@H]3CC[C@]2(C)O1</smiles>

17<smiles>Cc1cc(O)c([C@]2(C)CC[C@H]3CC3C2)cc1-c1cc([C@]2(C)CCC3CCC32C)c(C)cc1O</smiles>

19<smiles>C=C1C[C@H](O)[C@@H](Br)C(C)(C)[C@]12C=C/C(=C\Br)CC2</smiles>

22<smiles>C=C1CC(O)C(Br)C(C)(C)C12CC1C(Cl)CC(C)(Br)C1C2</smiles>

25

constituinte do extrato de L. majuscula coletada nas Filipinas, apresentou modesta atividade contra um painel de células do "National Cancer Institute" (NCI - Instituto Nacional do Câncer) (Erickson et al., 1995).

Alguns triterpenos apresentaram potente atividade citotóxica. Da espécie L. viridis coletada nas Ilhas Canárias, isolou-se uma série de triterpenos - deidrotirsiferol (11), deidrovenustatriol (12), isodeidrotirsiferol (13) e tirsenol B (14) - que apresentaram atividade citotóxica contra células P-388 com $\mathrm{IC}_{50}=0,01 \mu \mathrm{g} / \mathrm{mL}$ (Norte et al., 1996, 1997; Manriquez et al., 2001).

Da mesma espécie também foi possível isolar outros triterpenos: martiriol (15), pseudodeidrotirsiferol (16), dioxepandeidrotirsiferol (17) e 16-epihidroxideidrotirsiferol (18). Os seus efeitos citotóxicos foram avaliados mediante linhagens de células tumorais: P388 (neoplasma linfóide de camundongo), A-549 (carcinoma de pulmão), MEL28 (melanoma humano) e HT-29 (carcinoma de cólon humano). Nos testes realizados, o martiriol (15) foi inativo em concentrações inferiores a $10 \mu \mathrm{g} / \mathrm{mL}$, enquanto pseudodeidrotirsiferol (16), dioxepandeidrotirsiferol (17) e 16-epi-hidroxideidrotirsiferol (18) foram inativos em concentrações inferiores a $1 \mu \mathrm{g} / \mathrm{mL}$ (Manriquez et al.,<smiles>C=C(CC[C@@H](O)[C@]1(C)CC[C@@H](C(C)(C)O)O1)C1CCC2OC([C@H]3CC[C@H](C(C)O)O3)CC[C@]2(C)O1</smiles><smiles>C=C(C1CCC2OC(C3(C)CCC(Br)C(C)(C)O3)CC[C@]2(C)O1)[C@H](O)C[C@H](O)[C@]1(C)CC[C@H](C(C)(C)O)O1</smiles>

18<smiles>C=C1C[C@H](O)[C@H](Br)C(C)(C)C12CCC(C)=C(Cl)C2</smiles>

20

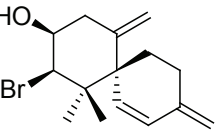

23<smiles>C=C1C[C@H](O)[C@@H](Br)[C@]2(C)CC/C(=C/Br)C=C[C@]12C</smiles>

21<smiles>C=C1C[C@H](O)[C@H](Br)[C@]2(C)C=CC(=O)CC[C@]12C</smiles>

24
2001).

Os efeitos citotóxicos de desidrotirsiferol (11) foram recentemente estudados em quatro linhagens de células de tumores de mama. Os resultados revelaram que a apoptose foi induzida de forma mais eficaz nas linhagens de células não responsivas a hormônios, consideradas mais invasivas e com maior potencial de produzir metástases quando comparadas às células hormônio-dependentes. Os autores consideram esta substância uma potencial candidata a fármaco para tratamento de tumores de mama (Pec et al., 2003).

Com o objetivo de estudar os metabólitos secundários de algas chinesas, Sun e colaboradores (2005) buscaram isolar os principais metabólitos de Laurencia tristicha. Durante a pesquisa, foram isolados quatorze sesquiterpenos, sendo que metade deles era inédita. Todas as substâncias foram testadas contra uma série de linhagens de células tumorais humanas, incluindo adenocarcinoma de pulmão (A549), câncer de estômago (BGC-823), hepatoma (Bel 7402), câncer de cólon (HCT-8) e linhagem de células HeLA. Apenas o sesquiterpeno não-halogenado laurebifenil (19) mostrou atividade moderada contra as linhagens testadas, com valores de $\mathrm{IC}_{50}$ que variaram entre 


\section{1,22 e $1,91 \mu \mathrm{g} / \mathrm{mL}$.}

Em 1997, Juagdan et al. publicaram os resultados de suas pesquisas com Laurencia cartilaginea. Desta alga, coletada no Havaí, foram isolados seis sesquiterpenos halogenados (20-25). Dentre eles o elatol (20), metabólito amplamente distribuído no gênero. As substâncias ma'ilione (24) e o alo-isobtusol (25), ainda não tinham sido isolados anteriormente. Todos os metabólitos apresentaram atividade marcante contra linhagens de células tumorais P-388, A-549, HT-29 (carcinoma de cólon humano) e MEL-28 em baixas concentrações, especialmente HT-29.

Extratos brutos metanólicos de espécimes de $L$. obtusa coletados no Japão exibiram atividade inibitória sobre o crescimento de células P388. O fracionamento deste extrato levou ao isolamento de diterpenos bromados. A avaliação da citotoxidade destes metabólitos revelou que apenas um destes é citotóxico (26) (Takeda et al., 1990).

Recentemente verificou-se a atividade citotóxica de 14 sesquiterpenos (27-40) obtidos de espécimes de $L$. catarinensis do litoral brasileiro contra as linhagens de células HT-29, MCF7 e A431. O sesquiterpeno 31 foi o mais potente em todos os ensaios e cinco das substâncias isoladas não apresentaram atividade em nenhuma linhagem testada $(\mathbf{2 9}, \mathbf{3 4}, \mathbf{3 6}, \mathbf{3 7}, \mathbf{3 8})$. Não foi possível correlacionar a atividade observada à presença ou ausência de grupos funcionais específicos.

Os dados de atividade citotóxica indicam que esta ação não parece estar concentrada em um grupo específico de metabólitos. Dependendo do padrão estrutural da série de metabólitos testados a presença de halogênios é ou não crucial para manutenção da atividade.

Apesar de existirem mais de 34 metabólitos testados com comprovada atividade citotóxica, somente os mecanismos de ação antitumoral para o diterpeno laurediterpineol (1) foram determinados.

\section{Atividade antibacteriana e antifúngica}

$\mathrm{O}$ rápido desenvolvimento de resistência na terapia antibacteriana e antifúngica tem motivado a pesquisa de novos fármacos capazes de combater de forma eficaz e segura estes patógenos. Vários estudos têm sido realizados com o objetivo de verificar a atividade antibacteriana e antifúngica dos metabólitos secundários de Laurencia (Bansemir, 2004). O resumo destes trabalhos é apresentado na Tabela 2.

Estudos feitos com bactérias marinhas apresentam relevância do ponto de vista das interações ecológicas. Mas, estas substâncias podem, eventualmente, atuar de forma equivalente em bactérias patogênicas humanas interferindo em mecanismos moleculares semelhantes.

Um dos estudos foi feito com a fração resultante da partição com acetato de etila do extrato metanólico de Laurencia obtusa coletada no México. A fração foi ativa contra as bactérias gram-positivas Staphylococcus aureus e Bacillus subtilis. Os testes com Trichophyton mentagrophytes revelaram atividade moderada contra este fungo filamentoso causador de dermatofitoses em unhas, cabelo e pele. Apesar de ser uma atividade moderada, este resultado é importante pela atual falta de antifúngicos eficientes e seguros no mercado. A fração não apresentou atividade contra bactérias gram-negativas (Escherichia coli, Pseudomonas aeruginosa, Klebsiella pneumoniae e Shigella flexneri), Streptococcus agalactiae e o fungo Aspergillus niger (Morales et al., 2006).

Foram realizados testes de atividade antibacteriana do extrato bruto de L. pannosa de uma amostra coletada na Malásia. O extrato foi analisado contra treze espécies de bactérias marinhas isoladas dos mares da Malásia. Observou-se atividade antibacteriana contra as bactérias Chromobacterium violaceum, Proteus mirabilis e Vibrio

Tabela 2. Atividade antibacteriana e antifúngica de metabólitos isolados do gênero Laurencia.

\begin{tabular}{|c|c|c|c|}
\hline Espécies & $\begin{array}{l}\text { Extratos, frações ou } \\
\text { substâncias }\end{array}$ & Organismos sensíveis & Referência \\
\hline \multirow[t]{3}{*}{ L. obtusa } & Fração acetato de etila & S. aureaus e B. subtilis & Morales et al., 2006 \\
\hline & & Trichophyton mentagrophytes & \\
\hline & 43 & $\begin{array}{l}\text { Bacillus megaterium } \\
\text { Ustilago violacea }\end{array}$ & Konig \& Wright, 1997 \\
\hline \multirow[t]{3}{*}{ L. pannosa } & Extrato bruto & Bactérias marinhas & Suzuki et al., 2001 \\
\hline & 41 & Bactérias marinhas & Suzuki et al., 2001 \\
\hline & 42 & Bactérias marinhas & Suzuki et al., 2001 \\
\hline L. yonaguniensis & 44 & Bactérias marinhas & Takahashi et al., 2002 \\
\hline \multirow[t]{5}{*}{ L. majuscula } & 45 & Bactérias marinhas & Vairappan et al., 2001a \\
\hline & 20,48 & Bactérias marinhas & Vairappan et al., 2001b \\
\hline & 20 & S. epidermis, K. pneumonia, Salmonella $\mathrm{sp}$. & Vairappan, 2003 \\
\hline & 48 & K. pneumonia, Salmonella sp. & Vairappan, 2003 \\
\hline & 43,50 & S. aureus, S. pneumoniae, E. faecalis, E. faecium, M. catarrhalis & Vairappan et al., 2004 \\
\hline L. nidifica & 46,47 & Bactérias marinhas & Vairappan et al., 2001a \\
\hline L. okamurae & 46,47 & S. aureus, S. pneumoniae, E. faecalis, E. faecium, M. catarrhalis & Vairappan et al., 2004 \\
\hline \multirow[t]{2}{*}{ L. glandulifera } & $51-54$ & S. aureus & Kladi et al., 2008 \\
\hline & & & $\begin{array}{r}\text { Rev. Bras. Farmacogn. } \\
\text { Braz. J. Pharmacogn. } \\
\text { 20(3): Jun./Jul. } 2010\end{array}$ \\
\hline
\end{tabular}


cholerae. A atividade antibacteriana dos sesquiterpenos panosanol (41) e panosano (42), isolados a partir deste extrato, foi avaliada frente a essas três bactérias. O panosanol, que é o principal metabólito desta espécie, apresentou atividade contra todas as bactérias testadas. A concentração mínima inibitória (CMI) foi de $60 \mu \mathrm{g} /$ disco contra $P$. mirabilis e $100 \mu \mathrm{g} /$ disco contra $C$. violaceum e $V$. cholerae. O panosano (42) apresentou atividade apenas nos ensaios com $C$. violaceum com CMI de $60 \mu \mathrm{g} / \mathrm{disco}$ (Suzuki et al., 2001).

O sesquiterpeno alolaurinterol (43) foi isolado com um rendimento de $33,5 \%$ a partir do extrato bruto de Laurencia obtusa coletada na ilha caribenha de Dominica. As atividades antifúngica e antibacteriana foram avaliadas. A ação mais proeminente foi observada contra a bactéria Bacillus megaterium e o fungo Ustilago violacea (Konig \& Wright, 1997).

Da espécie L. yonaguniensis encontrada no Japão, isolou-se um diterpeno inédito com esqueleto neoireano, chamado de neoirietetraol (44). Este metabólito foi testado por ensaios de difusão em disco para verificar atividade antibacteriana contra seis espécies de bactérias marinhas. O neoirietetraol mostrou-se ativo contra Alcaligenes aquamarinus e Escherichia coli em uma concentração de $100 \mu \mathrm{g} /$ disco (Takahashi et al., 2002).

Em um outro estudo utilizando amostras coletadas no Japão, isolou-se um sesquiterpeno bromado (45) de L. majuscula, que foi ativo contra cinco bactérias marinhas gram-negativas pertencentes a diferentes gêneros (Alcaligenes aquamarinus, Azomonos agilis, Azotobacter beijerinckii, Erwinia amyolovora e Escherichia coli), com CMI entre 10-30 $\mu \mathrm{g} /$ disco. Duas substâncias, laurinterol (46) e isolaurinterol (47) isoladas de L. nidifica, apresentaram melhores resultados nestes ensaios, com CMI entre $5-15 \mu \mathrm{g} /$ disco para as bactérias testadas (Vairappan et al., 2001a).

O mesmo grupo de pesquisas publicou simultaneamente um estudo com metabólitos isolados de amostras de Laurencia majuscula proveniente de cinco locais da Malásia. Os metabólitos majoritários nas amostras foram o elatol (20) e iso-obtusol (48). O elatol (20) apresentou atividade antibacteriana contra todas as treze bactérias marinhas testadas enquanto o iso-obtusol (48) apresentou atividade apenas frente a seis espécies (Vairappan et al., 2001b).

Baseado nestes resultados promissores frente a bactérias marinhas, Vairappan testou os efeitos dos metabólitos elatol (20) e iso-obtusol contra uma série de bactérias patogênicas humanas. O elatol (20) apresentou atividade antibacteriana contra Staphylococcus epidermis, Klebsiella pneumonia e Salmonella sp. Enquanto o isoobtusol (48) foi ativo contra $K$. pneumonia e Salmonella sp. Esse estudo comparou ainda estes dois metabólitos com uma série de quimioterápicos comercialmente disponíveis. Os estudos revelaram que o elatol (20) apresenta atividade igual ou até maior que alguns antibacterianos comercialmente disponíveis como o cefaclor e a canamicina (Vairappan, 2003).

Com o objetivo de estabelecer os potenciais dos metabólitos de Laurencia como protótipos para novos fármacos antibacterianos, uma série de sesquiterpenos halogenados foi submetida a ensaios contra 22 cepas de bactérias patogênicas humanas, incluindo sete cepas resistentes a antibióticos (Vairappan, 2004). O laurinterol (46) e isolaurinterol (47) foram isolados de L. okamurae. De L. majuscula coletada no Japão foram isolados alolaurinterol (43), contendo pequena quantidade de 10bromo-7-hidroxilaurano (49), e cupalaurenol (50).

Dos sesquiterpenos testados, o laurinterol (46), isolaurinterol (47), alolaurinterol (43) e cupalaurenol (50) apresentaram um amplo espectro de atividades contra bactérias gram-positivas, incluindo Staphylococcus aureus resistente à meticilina, Streptococcus pneumoniae resistente à penicilina e Enterococcus faecalis e E. faecium. As substâncias testadas só foram efetivas contra uma espécie de bactéria gram-negativa, Moraxella catarrhalis. As substâncias 43 e 46 apresentaram atividade bactericida contra $S$. aureaus resistente à meticilina em uma concentração de $6,25 \mu \mathrm{g} / \mathrm{mL}$, o que representa uma potência maior que a vancomicina. Estas substâncias apresentaram ação bactericida para E. faecium susceptível a vancomicina, enquanto a própria vancomicina apresenta ação bacteriostática.

O elatol (20) e iso-obtusol (48) isolados de $L$. majuscula de vários locais da Malásia também foram investigados, mas não apresentaram atividade.

$\mathrm{O}$ fato dos metabólitos 20 e $\mathbf{4 8}$ não terem apresentado bons resultados nesta pesquisa pode revelar o desenvolvimento de resistência bacteriana. Mas ainda assim, os dados publicados indicam que os sesquiterpenos halogenados são potenciais fármacos na luta contra bactérias resistentes a vários quimioterápicos.

Uma série de acetogeninas isoladas de $L$. glandulifera, coletada na Ilha de Creta, foi submetida a ensaios de atividade antibacteriana contra um painel de cepas de Staphylococcus aureaus multi-resistentes. As acetogeninas 51-54 apresentaram concentração mínima inibitória na faixa de $8-256 \mu \mathrm{g} / \mathrm{mL}$. A presença dos grupamentos acetila em $\mathbf{5 2}$ desempenha um papel crucial na atividade observada, provavelmente por melhorar a biodisponibilidade celular através da maior lipofilicidade (Kladi et al., 2008).

\section{Atividade antiparasitária}

As doenças parasitárias são freqüentemente relatadas como negligenciadas pelas grandes empresas farmacêuticas e instituições de países desenvolvidos. Como a pesquisa nesta área se concentra em países em desenvolvimento, o número de trabalhos nesta área ainda é restrito.

A análise fitoquímica do extrato bruto de 


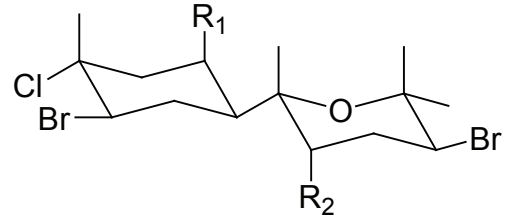

$27 \mathrm{R}_{1}=\mathrm{OAc}, \mathrm{R}_{2}=\mathrm{OH}$

$28 \mathrm{R}_{1}=\mathrm{OH}, \mathrm{R}_{2}=\mathrm{OAC}$

$29 \mathrm{R}_{1}=\mathrm{OAc}, \mathrm{R}_{2}=\mathrm{OAc}$

$30 \mathrm{R}_{1}=\mathrm{OAc}, \mathrm{R}_{2}=\mathrm{H}$

$31 \mathrm{R}_{1}=\mathrm{H}, \mathrm{R}_{2}=\mathrm{OH}$

$32 \mathrm{R}_{1}=\mathrm{H}, \mathrm{R}_{2}=\mathrm{OAC}$

$33 \mathrm{R}_{1}=\mathrm{R}_{2}=\mathrm{H}$

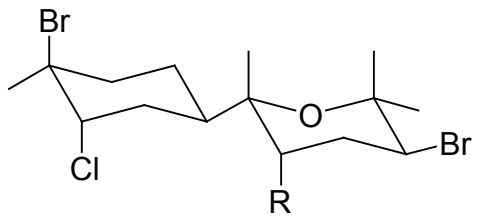

$38 \mathrm{R}=\mathrm{OH}$

$39 \mathrm{R}=\mathrm{H}$<smiles>C=C1[C@H](C)CC[C@@](C)(O)[C@]12CC[C@@](C)(Cl)[C@H](Br)C2</smiles>

41

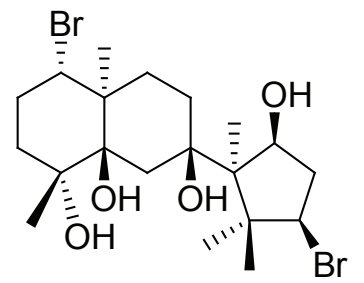

44

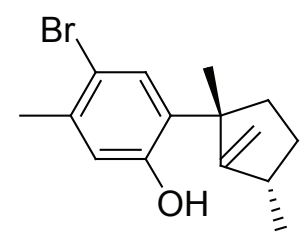

47

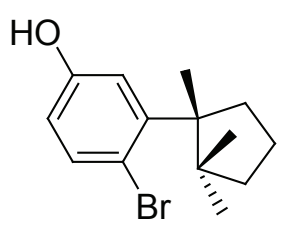

50

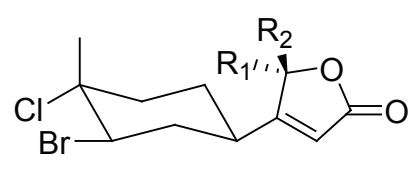

$34 \mathrm{R}_{1}=\mathrm{Me}, \mathrm{R}_{2}=\mathrm{OMe}$

$35 \mathrm{R}_{1}=\mathrm{OMe}, \mathrm{R}_{2}=\mathrm{Me}$

$36 \mathrm{R}_{1}=\mathrm{Me}, \mathrm{R}_{2}=\mathrm{OH}$

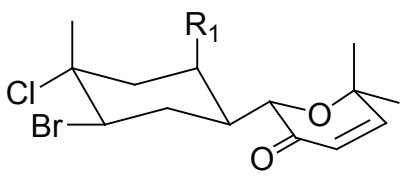

40

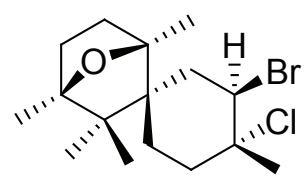

42<smiles>C=C1C[C@H](O)[C@@H](Br)C(C)(C)[C@]12CC=C(C)CC2</smiles>

45

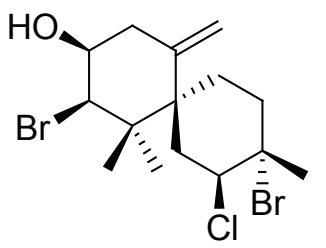

48

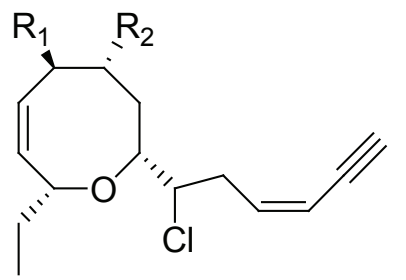

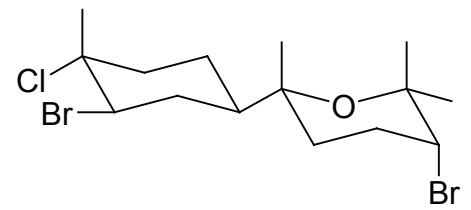

37

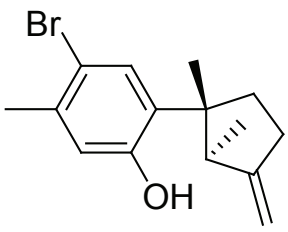

43

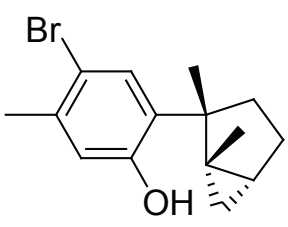

46

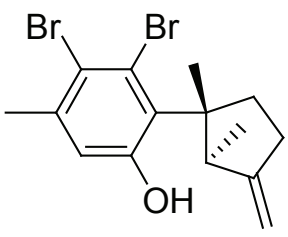

49

$51 \mathrm{R}_{1}=\mathrm{R}_{2}=\mathrm{OH}$

$52 \mathrm{R}_{1}=\mathrm{R}_{2}=\mathrm{OAC}$

$53 \mathrm{R}_{1}=\mathrm{OAc} ; \mathrm{R}_{2}=\mathrm{OH}$

$54 \mathrm{R}_{1}=\mathrm{OH} ; \mathrm{R}_{2}=\mathrm{OAc}$ 
Laurencia obtusa coletada na Turquia resultou no isolamento de um novo sesquiterpeno $\mathbf{5 5}$ capaz de inibir $90 \%$ do crescimento da cepa D6 sensível à cloroquina de Plasmodium falciparum. A partir destes resultados, foram feitos estudos de dose-resposta com cepas D6 e W2, esta última resistente à cloroquina. $\mathrm{O}$ metabólito apresentou atividade moderada com valores de $\mathrm{IC}_{50}$ de 2700 e 4000 $\mathrm{ng} / \mathrm{mL}$ contra os clones D6 e W2, respectivamente (Topcu et al., 2003).

Os extratos etanólicos de L. obtusa e $L$. corallopsis apresentaram atividade inibidora in vitro sobre a cepa de Plasmodium falciparum F32/Tanzânia. A concentração mínima inibitória variou de 44 a $162 \mu \mathrm{g} /$ $\mathrm{mL}$. Estes valores não são inferiores ao observado para a cloroquina (100 ng/mL) (Martínez et al., 2003). Um estudo publicado recentemente revelou que o extrato de $L$. microcladia apresenta atividade promissora contra a forma promastigota de Leishmania mexicana (Freile-Pelegrin et al., 2008). Dados recentes revelam que o sesquiterpeno elatol (20) apresenta ação frente ao Trypanosoma cruzi. (Veiga-Santos et al., 2008)

A tricomoníase é uma doença sexualmente transmissível causada pelo protozoário Trichomonas vaginalis que infecta o trato urogenital de homens e mulheres. Em um estudo de triagem de atividade tricomonicida com diversas algas, o extrato de $L$. microcladia apresentou um dos melhores resultados entre as algas vermelhas com $\mathrm{IC}_{50}$ de 4,9 $\pm 0,5 \mu \mathrm{g} / \mathrm{ml}$ (Moo-Puc et al., 2008).

A atividade anti-helmíntica de uma série de metabólitos isolados de Laurencia scoparia (56-63) coletada no Brasil revelou uma atividade moderada contra o estágio L4 de Nippostrongylus brasiliensis (modelo in vitro). Entretanto, nenhuma das substâncias testadas foi mais potente que o fármaco utilizado como referência, albendazol (Davyt, 2001). Posteriormente, foi relatado o isolamento de um sesquiterpeno do tipo $\beta$-bisaboleno 64 a partir da mesma espécie, proveniente do mesmo local de coleta. Esta substância também apresentou atividade antiparasitária moderada contra o estágio L4 de $N$. Brasiliensis (Davyt et al., 2006).

\section{Atividade antiviral}

O número de fármacos antivirais comercialmente disponíveis ainda está distante da necessidade real de substâncias eficazes no combate de uma gama variada de vírus causadores de diversas patologias, como a dengue, febre amarela, hepatite e AIDS. Um grande número de substâncias com atividade antiviral isoladas de algas marinhas vem sendo descrito recentemente (Mayer et al., 2007).

Na década de 80, Sakemi e colaboradores (1986), buscando moléculas bioativas a partir de organismos marinhos da costa do Japão, verificaram que o extrato bruto de Laurencia venusta apresentava atividade contra os vírus da estomatite vesicular (VSV) e herpes simplex tipo 1. O fracionamento, guiado por bioensaio, levou ao isolamento de três substâncias ativas, identificadas como tirsiferol (65), 23-acetato de tirsiferol e venustratriol (66).

Iliopoulou e colaboradores (2002b) isolaram cinco sesquiterpenos inéditos e outros metabólitos conhecidos (67-74) de Laurencia obtusa coletada na Grécia. Essas substâncias foram avaliadas frente aos vírus HIV, herpes simplex 1 e 2, vaccinia, estomatite vesicular, respiratório sincicial, parainfluenza 3 e renovírus. Entretanto, nenhuma das substâncias testadas apresentou atividade significativa contra qualquer dos vírus testados.

Apesar de serem poucos os estudos publicados sobre atividade antiviral para metabólitos produzidos pelo gênero Laurencia, os dados atuais sugerem que esta ação pode estar restrita aos triterpenos produzidos pelo gênero.

\section{Atividade analgésica e anti-asmática}

O diterpeno tricíclico bromado neorogioltriol(75), isolado de $L$. glandulifera, na dose de $1 \mathrm{mg} / \mathrm{kg}$ (injeção intraperitonial) foi eficiente em reduzir o número de contorsões induzidas por ácido acético em ratos em 88,9\%. O ácido acetilsalicílico, um famoso inibidor da produção de prostaglandinas, apresenta um efeito similar em uma dose de $200 \mathrm{mg} / \mathrm{kg}$.Os autores propõem que o mecanismo de ação deste diterpeno envolve a inibição de mediadores da inflamação, como prostaglandinas, sugerindo que a molécula 75 é um antagonista da ciclooxigenase ou de outras enzimas da cascata do ácido araquidônico (Chatter et al., 2009).

O extrato etanólico de L. undulata, rico em polifenóis, inibiu a hiper-reatividade das vias respiratórias e a inflamação induzidas por ovalbumina em um modelo murino de asma. O resultado indica que este extrato poderá ser utilizado como adjuvante na terapia da asma brônquica (Won-Kyo et al., 2009).

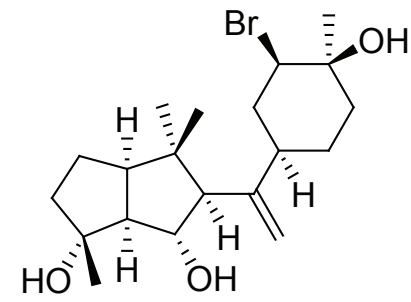

75

Do extrato de L. undulata isolou-se dois açúcares: floridosídeo (76) e D-isofloridosídeo (77) e verificou-se a ação antioxidante destas substâncias através de ensaios de inibição de espécies reativas de oxigênio e aumento na expressão dos genes glutationa e superóxido dismutase. Os resultados obtidos sugerem que 76 e 77 podem ser responsáveis pelas ações farmacológicas do extrato bruto de L. undulata (Yong-Xin et al. 2010). 
<smiles>C=CC(C)(O)CC[C@H]1C(=C)C(Br)C[C@@H](Br)C1(C)C</smiles>

55<smiles>C=C1C[C@H](O)C(Br)[C@@]2(C)C=CC(=O)CCC12</smiles>

59<smiles>C=C1CC[C@H](Br)C(C)(C)[C@]12CC[C@@](C)(Br)[C@@H](Cl)C2</smiles>

62

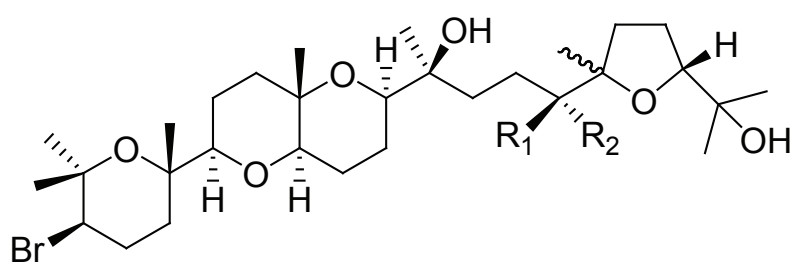

$65 \mathrm{R}_{1}=\mathrm{OH}, \mathrm{R}_{2}=\mathrm{H}, \mathrm{C} 29=\alpha \mathrm{CH}_{3}$

$66 \mathrm{R}_{1}=\mathrm{H}, \mathrm{R}_{2}=\mathrm{OH}, \mathrm{C} 29=\beta \mathrm{CH}_{3}$<smiles>[R]C1C(=O)[C@H](C)[C@]23CC[C@]2(C)[C@@H](Br)C[C@]3(C)[C@@H]1C</smiles>

$$
\begin{aligned}
& 69 \mathrm{R}=\mathrm{Br} \\
& 70 \mathrm{R}=\mathrm{OCH}_{3} \\
& 71 \mathrm{R}=\mathrm{H}
\end{aligned}
$$<smiles>[R]C1C(=O)C(C)=C2CCC(C)=CC[C@]2(C)[C@H]1C</smiles>

$72 \mathrm{R}=\mathrm{OCH}_{3}$ $73 \mathrm{R}=\mathrm{H}$<smiles>[R]C([R4])=C1C=C[C@]2(CC1)C(=C)C[C@@H](O)[C@H](Br)C2(C)C</smiles>

$57 \mathrm{R}_{1}=\mathrm{H} ; \mathrm{R}_{2}=\mathrm{Br}$

$58 \mathrm{R}_{1}=\mathrm{Br} ; \mathrm{R}_{2}=\mathrm{H}$<smiles>C=C1C[C@H](O)[C@H](Br)C(C)(C)[C@]12C=CC(C)=CC2</smiles>

61<smiles>C=C(C[C@H](O)C=C(C)C)[C@]1(O)CC[C@@](C)(Br)[C@H](Cl)C1</smiles>

64<smiles>[R]C1C(=O)[C@H](C)[C@@]2(C)[C@@H](C)C[C@@]3(C)C[C@@]12C[C@@H]3Br</smiles>

$67 \mathrm{R}=\mathrm{Br}$ $68 \mathrm{R}=\mathrm{H}$<smiles>CC1=C2CC[C@](C)(Cl)[C@@H](Br)C[C@]2(C)[C@H](C)[C@@H](Br)[C@@H]1O</smiles><smiles>OCC(CO)OC1CC(CO)C(O)C(O)C1O</smiles>

76

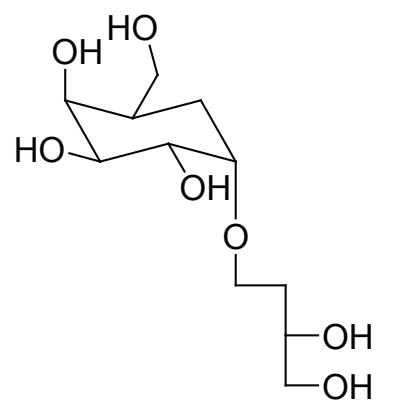

77 


\section{CONCLUSÕES}

Apesar do grande número de metabólitos já isolados para o gênero Laurencia, a grande maioria ainda não foi submetida a ensaios de atividade farmacológica.

Das 145 espécies descritas até o momento, apenas são encontrados estudos de atividade biológica para os metabólitos isolados de pelo menos quinze delas, o que corresponde a aproximadamente $10 \%$ do total. Dentre os metabólitos descritos, cerca de 65 moléculas já foram avaliadas quanto à atividade farmacológica, principalmente em testes para avaliação de citotoxicidade e atividade antibacteriana. As moléculas ativas, na sua grande maioria, são halogenadas, contendo principalmente o átomo de bromo em sua estrutura. Do total de moléculas avaliadas, 34 moléculas apresentaram atividade citóxica, quinze apresentaram atividade antibacteriana, duas apresentaram atividade antiviral e onze moléculas apresentaram atividade antiparasitária, além de três diferentes extratos lipofílicos ativos. A presença de átomos de halogênios em praticamente todas as moléculas ativas sugere que a presença desses elementos pode estar relacionada aos efeitos observados, principalmente em relação aos ensaios de atividade antibacteriana. Nesses casos, todas as moléculas ativas são halogenadas. Quando são observados os testes de atividade citotóxica e antiviral, o maior número de moléculas ativas contém halogênios, porém, as ações nesses casos não parecem estar restritas a esse tipo de funcionalização. A Figura 1 apresenta a distribuição das diferentes atividades para os metabólitos descritos para o gênero Laurencia.

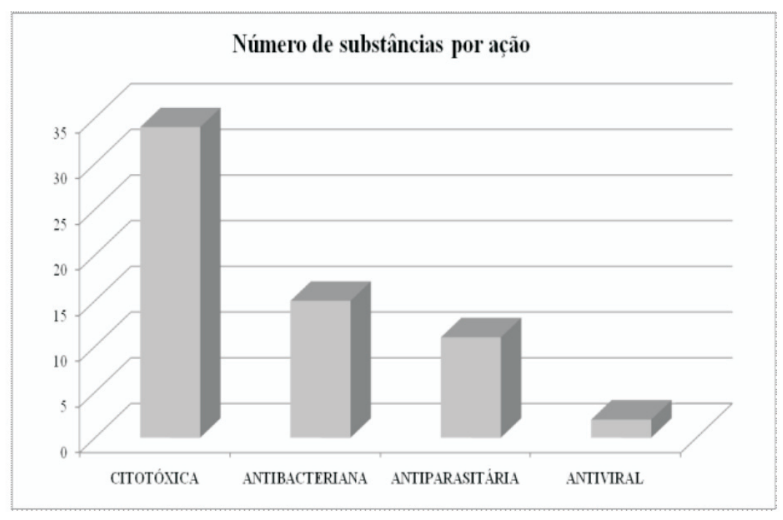

Figura 1. Distribuição dos metabólitos secundários de Laurencia por atividade farmacológica.

Como pode ser observado, os resultados de atividade farmacológica estão concentrados na área de oncologia, com base nos estudos de avaliação da atividade citotóxica frente a uma série de células tumorais. Este fato pode estar relacionado ao grande interesse de grupos de pesquisas no mundo inteiro trabalhando nesta área, além da existência de grandes centros como "National Cancer Institute" dos Estados Unidos, cujo foco principal é o entendimento dos mecanismos moleculares presentes no câncer e a busca de novos fármacos antitumorais.

$\mathrm{O}$ número de substâncias com atividade antibacteriana também é elevado, principalmente devido ao grande número de estudos ecológicos realizados. Entretanto, o número de moléculas ativas frente a bactérias patogênicas humanas ainda é pequeno. Os resultados já observados, aliados à diversidade de metabólitos disponíveis, indicam claramente que a pesquisa de metabólitos produzidos pelo gênero Laurencia, - amplamente distribuído no nosso litoral - possui um grande potencial de obtenção de novos protótipos de fármacos a serem utilizados no tratamento de diversas patologias.

\section{AGRADECIMENTOS}

Os autores agradecem à FAPERJ, FINEP e ao CNPq pelo suporte financeiro. FLSM agradece à CAPES pela bolsa de mestrado. Os autores agradecem a Heitor M. Duarte pela valiosa revisão.

\section{REFERÊNCIAS}

Algaebase. Disponível em: <www.algaebase. org $>$, acessado em junho de 2008. Algamare 2008. Macroalgas marinhas da costa brasileira - estado do conhecimento, usos e conservação biológica. Disponível em: <http://www.ib.usp.br/ algamare-br/menu.htm>, acessado em junho de 2008 .

BansemirA,JustN,MichalikM,LindequistU,LalkM2004.Extracts and sesquiterpene derivatives from the red alga Laurencia chondrioides with antibacterial activity against fish and human pathogenic bacteria. Chem Biodiver 1: 463-467.

Blunt JW, Copp BR, Hu WP, Munro MHG, Northcote, PT, Prinsep MR2007. Marinenatural products. NatProdRep 24:31-86.

Cardozo KHM, Guaratini T, Barros MP, Falcão VR, Tonon AP, Lopes NP, Campos S, Torres MA, Souza AO, Colepicolo P, Pinto E 2007. Metabolites from algae with economical impact. Comp Biochem Physiol Part C 146: 60-78.

Carter-Franklin JN, Butler A 2004. Vanadium bromoperoxidasecatalyzed biosynthesis of halogenated marine natural products. J Am Chem Soc 126: 15060-15066.

Chatter R, Kladi M, Tarhouni S, Maatoug R, Kharrat R, Vagias C, Roussis V 2008. Neorogioltriol: a brominated diterpene with analgesic activity from Laurencia glandulifera. Phytochemistry Lett 2: 25-28.

Davyt D, Fernandez R, Suescun L, Mombru AW, Saldana J, Dominguez L, Coll J, Fujii MT, Manta E 2001. New sesquiterpene derivatives from the red alga Laurencia scoparia. Isolation, structure determination, and anthelmintic activity. $J$ Nat Prod 64: 1552-1555.

Davyt D, Fernandez R, Suescun L, Mombru AW, Saldana J, Dominguez L, Fujii MT, Manta E 2006. Bisabolanes from the red alga Laurencia scoparia. JNat Prod 69: 1113-1116.

De Carvalho LR, Fujii MT, Roque NF, Lago JHG 2006. Aldingenin derivatives from the red alga Laurencia aldingensis. Phytochemistry 67: 1331-1335.

Dixon N, Wong LS, Geerlings TH, Micklefield J 2007. Cellular targets of natural products. Nat Prod Rep 24: 1288-1310.

Erickson KL, Beutler JA, Gray GN, Cardellina JH, Boyd 
MR 1995. Majapolene A, a cytotoxic peroxide, and related sesquiterpenes from the red alga Laurencia majuscula. J Nat Prod 58:1848-1860.

Freile-Pelegrin Y, Robledo D, Chan-Bacab MJ, OrtegaMorales BO 2008. Antileishmanial properties of tropical marine algae extracts. Fitoterapia 79: 374-377.

Iliopoulou D, Vagias C, Harvala C, Roussis V 2002a. C15 acetogenins from the red alga Laurencia obtusa. Phytochemistry 59: 111-116.

Iliopoulou D, Roussis V, Pannecouque C, De Clercq E, Vagias C 2002b. Halogenated sesquiterpenes from the red alga Laurencia obtusa. Tetrahedron 58: 6749-6755.

Iliopoulou D, Mihopoulos N, Vagias C, Papazafiri P, Roussis V 2003. Novel cytotoxic brominated diterpenes from the red alga Laurencia obtusa. J Org Chem 68: 7667-7674.

Irie T, Suzuki M, Masamune T 1965. Laurencin, a constituent from Laurencia species. Tetrahedron Lett 6: 1091-1099.

Juagdan EG, Kalidindi R, Scheuer P 1997. Two new chamigranes from an Hawaiian red alga Laurencia cartilaginea. Tetrahedron 53:521-528.

Kladi M, Vagias C, Furnari G, Moreau D, Roussakis C, Roussis $\mathrm{V}$ 2005. Cytotoxic cuparene sesquiterpenes from Laurencia microcladia. Tetrahedron Lett 46: 5723-5726.

Kladi M, Xenaki H, Vagias C, Papazafiri P, Roussis $\mathrm{V}$ 2006. New cytotoxic sesquiterpenes from the red algae Laurencia obtusa and Laurencia microcladia. Tetrahedron 62:182-189.

KladiM,VagiasC,StavriM, RahmanM, Gibbons S, Roussis V2008. $\mathrm{C} 15$ acetogenins with antistaphylococcal activity from the red alga Laurencia glandulifera. Phytochem Lett 1:31-36.

Konig GM, Wright AD, Sticher O, Angerhofer CK, Pezzuto JM 1994. Biological-activities of selected marine natural-products. Planta Med 60:532-537.

Konig G M, Wright AD 1997. Sesquiterpene content of the antibacteirial dichloromethane extract of the marine red alga Laurencia obtusa. Planta Med 63: 186-187.

Lhullier C, Falkenberg M, Ioannou E, Quesada A, Papazafiri P, Horta PA, Schenkel EP, Vagias C, Roussis V 2010. Cytotoxic halogenated metabolites from the Brazilian Red Alga Laurencia catarinensis. J Nat Prod 73: 27-32.

Mayer AMS, Rodríguez AD, Berlinck RGS, Hamann MT 2007. Marine pharmacology in 2003-4: Marine compounds with anthelmintic antibacterial, anticoagulant, antifungal, anti-inflammatory, antimalarial, antiplatelet, antiprotozoal, antituberculosis, and antiviral activities; affecting the cardiovascular, immune and nervous systems, and other miscellaneous mechanisms of action. Comp Biochem Physiol Part C 145: 553-581.

Manriquez CP, Souto ML, Gavin JA, Norte M, Fernandez JJ 2001. Several new squalene derived triterpenes from Laurencia. Tetrahedron 57: 3117-3123.

Martínez JM, Hernández H, Acuña D, Esquivel M, Lizama RS, Payrol JA 2003. Actividad inhibidora del crecimiento in vitro de Plasmodium falciparum de extractos de algas del género Laurencia. Rev Cub Med Trop [online] 57 (3). Disponível em http://scielo. sld.cu/scielo.php?script $=$ sci_arttext\&pid $=$ S0375$07602005000300005 \& \overline{1} \mathrm{~g}=\mathrm{es} \& \mathrm{nrm}=$ is o, acessado em 22 de maio de 2010.

Mohammed KA, Hossain CF, Zhang L, Bruick RK, Zhou YD, Nagle DG 2004. Laurenditerpenol, a new diterpene from the tropical marine alga Laurencia intricata that potently inhibits HIF-1 mediated hypoxic signaling in breast tumor cells. J Nat Prod 67: 2002-2007. Molinski TF, Dalisay DS, Lievens SL, Saludes JP 2009. Drug development from marine natural products. Nat Rev Drug Disc 8: 69-85.

Moo-Puc R, Robledo D, Freile-Pelegrin Y 2008. Evaluation of selected tropical seaweeds for in vitro antitrichomonal activity. $J$ Ethnopharmacol 120: 82-97.

Morales JL, Cantillo-Ciau ZO, Sanchez-Molina I, MenaRejon GJ 2006. Screening of antibacterial and antifungal activities of six marine macroalgae from coasts of Yucatan peninsula. Pharm Biol 44: 632-635.

Norte M, Fernandez JJ, Souto ML, Garcia-Gravalos MD 1996. Two new antitumoral polyether squalene derivatives. Tetrahedron Lett 37: 2671-2674.

Norte M, Fernandez JJ, Souto ML, Garcia-Gravalos MD 1997. Thyrsenols A and B, two unusual polyether squalene derivatives. Tetrahedron 53: 3173-3178.

Pereira RC, Da Gama BA, Teixeira VL, Yoneshigue-Valentin Y 2003. Ecological roles of natural products of the Brazilian red seaweed Laurencia obtusa. Braz J Biol 63: 665-672.

Pec MK, Aguirre A, Moser-Their K, Fernandez JJ, Souto ML, Dorta J, Diaz-Gonzalez F, Villar J 2003. Induction of apoptosis in estrogen dependent and independent breast cancer cells by the marine terpenoid dehydrothyrsiferol. Biochem Pharmacol 65: 1451-1461.

Rochfort SJ, Capon RJ 1996. Parguerenes revisited: new brominated diterpenes from the southernAustralian marine red alga Laurencia filiformis. Aust J Chem 49: 19-26.

Rashid MA, Gustafson KR, Cardellina JH, Boyd MR 1995. Brominated chamigrane sesquiterpenes produce a novel profile of differential cytotoxicity in the NCI in-vitro screen. Nat Prod Lett 6: 255-259.

Sakemi S, Higa T, Jefford CW, Bernardinelli G 1986. Venustatriol - a new, antiviral, triterpene tetracyclic ether from Laurencia venusta. Tetrahedron Lett 27: 4287-4290.

Smit AJ 2004. Medicinal and pharmaceutical uses of seaweed natural products: A review. J Appl Phycol 16: 245-262.

Souto ML, Manriquez CP, Norte M, Fernandez JJ 2002. Novel marine polyethers. Tetrahedron 58: 8119-8125.

Sun J, Han LJ, Shi DY, Fan X, Wang SJ, Li S, Yang YC, Shi JG 2005. Sesquiterpenes from the red alga Laurencia tristicha. $J$ Nat Prod 68: 915-919.

Suzuki M, Daitoh M, Vairappan CS, Abe T, Masuda M 2001. Novel halogenated metabolites from the Malaysian Laurencia pannosa. J Nat Prod 64: 597-602.

Takahashi Y, Daiton M, Suzuki M, Abe T, Masuda M 2002. Halogenated metabolites from the new Okinawan red alga Laurencia yonaguniensis. J Nat Prod 65: 395-398.

Takeda S, Kurosawa E, Komiyama K, Suzuki T 1990. Constituents of marine plants. The structures of cytotoxic diterpenes containing bromine from the Marine Red Alga Laurencia obtusa (Hudson) Lamouroux. Bull Chem Soc Japan 63: 3066-3072.

Topcu G, Aydogmus Z, Imre S, Goren AC, Pezzuto JM, Clement JA, Kingston DGI 2003. Brominated sesquiterpenes from the red alga Laurencia obtusa. J Nat Prod 66: 1505-1508.

Vairappan CS, Suzuki M, Abe T, Masuda M 2001a. Halogenated metabolites with antibacterial activity from the Okinawan Laurencia species. Phytochemistry 58: 517-523.

Vairappan CS, Daitoh M, Suzuki M, Abe T, Masuda M 2001b. Antibacterial halogenated metabolites from the Malaysian Laurencia species. Phytochemistry 58: 291-297. Vairappan CS 2003. Potent antibacterial activity of halogenated 
metabolites from Malaysianredalgae Laurenciamajuscula (Rhodomelaceae, Ceramiales). Biomol Eng 20: 255-259.

Vairappan CS, Kawamoto T, Miwa H, Suzuki M 2004. Potent antibacterial activity of halogenated compounds against antibiotic-resistant bacteria. Planta Med 70: 1087-1090.

Veiga-Santos P, Rocha KJP, Dos Santos AO, Ueda-Nakamura T, Filho BPD, Lautenschlager SOS, Sudati DB, Bianco EM, Pereira RC, Nakamura, CV 2008. Antitrypanosomal activity of elatol isolated from red seaweed Laurencia obtusa. Planta Med 74: PA122.

Yong-Xin L, Yong L, Sang-Hoon L, Zhong-Ji Q, Se-Kwon K 2010. Inhibitors of oxidation and matrix metalloproteinases, floridoside and D-isofloridoside from marine red alga Laurencia undulata. J Agric Food Chem 58: 578-586.

Won-Kyo J, Inhak C, Sangtaek O, Sae-Gwang P, Su-Kil S, SooWoong L, Dae-Sung L, Soo-Jin H, You-Jin J, Jae-Young J, Chang-BumA, Jin SK, Kwang SO, Young-Mog K, Chisook M, Il-Whan C 2009. Anti-asthmatic effect of marine red alga (Laurencia undulata) polyphenolic extracts in a murine model of asthma. Food Chem Toxicol 47: 293-297.

Wright AD, Konig G M, Sticher O 1991. New sesquiterpenes and $\mathrm{C} 15$ acetogenins from the marine red alga Laurencia implicata. J Nat Prod 54: 1025-1033. 